\title{
Targeting facial aging with nano and regenerative technologies and procedures
}

\author{
Marek Dobke, Adam Hauch \\ Division of Plastic and Reconstructive Surgery, Department of Surgery, University of California San Diego, San Diego, CA 92103, USA.
}

Correspondence to: Prof. Marek Dobke, Division of Plastic and Reconstructive Surgery, Department of Surgery, UC San Diego, 200 West Arbor Drive, San Diego, CA 92103-8890, USA. E-mail: mdobke@health.ucsd.edu

How to cite this article: Dobke M, Hauch A. Targeting facial aging with nano and regenerative technologies and procedures. Plast Aesthet Res 2020;7:1. http://dx.doi.org/10.20517/2347-9264.2019.65

Received: 30 Nov 2019 First Decision: 2 Jan 2020 Revised: 2 Jan 2020 Accepted: 7 Jan 2020 Published: 16 Jan 2020

Science Editors: John Yousif, Kai O. Kaye Copy Editor: Jing-Wen Zhang Production Editor: Tian Zhang

\begin{abstract}
This study reviews information about the most novel ideas and modalities being incorporated into facial and neck cosmetic care. We seek to identify trends and developments within these areas as well as perceptions of plastic surgeons regarding their probable significance, and discussion on how these modalities may impact future practice patterns. It is hypothesized that nano and regenerative technologies are considered the most "hopeful". Emerging invasive and non-invasive modalities utilizing nano and regenerative concepts were reviewed. We intentionally sought to investigate approaches to beautification, including maintenance and reversal of signs of aging, utilizing methods lacking an established level of evidence. This included promising modalities which are currently at the investigational stage. Twelve board-certified plastic surgeons were surveyed regarding the clinical importance of twelve concepts and their expected impact on facial and neck rejuvenation. Scientific and technological creativity in aesthetics is rapidly changing, and the efficacy of innovations and safety margins are improving. Nano and regenerative medicine-based technologies and procedures were ranked most promising for the future of cosmetics. Their potential applications and research were reviewed in the context of surgical and non-surgical modalities in clinical practice. There has been an advent of new approaches to facial and neck aesthetic surgery and tissue care, which is well-beyond just skin care. With this new level of knowledge regarding variability in care responses and indications for procedures on an almost "molecular level", personalized and precise aesthetic surgery and medicine are quickly becoming a reality.
\end{abstract}

Keywords: Face, neck rejuvenation, nano, regenerative technologies

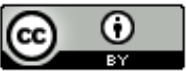

(C) The Author(s) 2020. Open Access This article is licensed under a Creative Commons Attribution 4.0 International License (https://creativecommons.org/licenses/by/4.0/), which permits unrestricted use, sharing, adaptation, distribution and reproduction in any medium or format, for any purpose, even commercially, as long as you give appropriate credit to the original author(s) and the source, provide a link to the Creative Commons license, and indicate if changes were made.

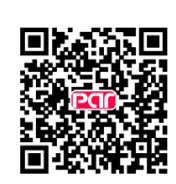




\section{INTRODUCTION}

Many technologies are currently available for developing effective cosmeceuticals with the goals of facial rejuvenation through procedures with varying levels of "invasiveness". These include non-invasive and invasive (requiring anesthesia) modalities; multi-platform devices for tissue contouring, comprising skin tightening modalities, fat reduction, and/or volumization procedures; genomic/molecular-level intervention to slow down the aging process; and nano and regenerative technologies ${ }^{[1,2]}$. Molecular concepts in the field of cosmetology indicate that even seemingly distant human body components and parameters such as gut microbiome and skin quality are related ${ }^{[3]}$. Furthermore, nanorobots and/or specific nanocosmeceuticals may form micellar nanostructures capable of carrying both hydrophilic and hydrophobic agents with rapid uptake into cells. These agents can be, for example, antibiotics to treat acne or agents targeting interventions on the molecular level such as prevention of telomere attrition, thus protecting skin DNA and structural proteins from aging and loss of native growth factors ${ }^{[1,4]}$.

The goal of this review is to present and discuss current and potential future trends in facial and neck rejuvenation. In doing so, this review links current technologies with what may be considered somewhat futuristic approaches and perspectives. The authors felt that it was important to survey practicing boardcertified plastic surgeons to determine how they felt about the significance of emerging trends (not only nano and regenerative), their expected prevalence and applicability to the areas of facial and neck aesthetic surgery practices in the coming decade, and to determine whether the perspective of scientific researchers is congruent with non-academic practitioners. Furthermore, the authors sought to potentially determine educational priorities among plastic surgeons.

From this analysis, the question that arises is whether the familiarity with nano and regenerative concepts is useful to a practitioner. The answer should be "yes", as trends clearly indicate that many procedures may be combined and include approaches which may appear to be distant and esoteric today, but are clearly going to be a large part of the daily practice of facial rejuvenation in the near future. Lastly, the question arises as to why to review nanoscience and regenerative technologies in one article. The simple reason is that it appears that both are tightly intermingled and one of the primary goals of this review is to point out and comment on these links ${ }^{[2,5]}$.

\section{DEFINITIONS}

For the purpose of this review, the following definition of nanotechnology was applied: nanoscience is the area of science, including healthcare, that deals with developing and producing extremely small devices and molecules. Nanoscale materials are classified in the range of $10^{-9} \mathrm{~m}$ (there are $25,400,000$ nanometers in an inch $)^{[6]}$.

Regeneration is defined as the process of renewal, restoration, and regrowth of damaged tissue or tissue replaced in the process of natural fluctuation or events (e.g., hair). Regeneration can be either complete, where the new tissue is the same as the tissue replaced, or incomplete, where different qualities of tissue replace the original (e.g., scar formation). Aspects of the regenerative processes for nerves, bones, and skin were traditionally emphasized and studied in plastic surgery. Advances in molecular biology and empiric clinical observations provided a basis for the development of interventions utilizing the potential regenerative properties of stem cells obtained from adipose tissue and perivascular tissue components ${ }^{[2,7,8]}$.

\section{SAMPLING PLASTIC SURGEONS' VISIONS REGARDING TRENDS IN FACIAL PLASTIC SURGERY}

A review of the literature was conducted using the following keywords as search criteria: nanotechnology, nanocosmeceuticals, and regenerative procedures for facial and neck rejuvenation. The results of this 
Table 1. Survey of plastic surgeons regarding modalities impacting the practice of facial and neck rejuvenation

\begin{tabular}{|c|c|}
\hline Modality rank & Score \\
\hline 1. Non-invasive stem cell and regenerative approaches & 135 \\
\hline $\begin{array}{l}\text { 2. Molecular level interventions, e.g., prevention of telomeres attrition protecting skin DNA and structural proteins from aging, } \\
\text { from loss of native growth factors }\end{array}$ & 119 \\
\hline 3. Improvements in percutaneous delivery of cosmeceuticals, personalized cosmetics & 115 \\
\hline 4. Genomic interventions & 109 \\
\hline 5. Tissue contouring/repair by energy delivering modalities & 100 \\
\hline 6. Refinements in body shaping by surgical techniques including tissue fllers and scaffolds & 96 \\
\hline 7. Skin resurfacing, tightening techniques (light, lasers) & 69 \\
\hline 8. Nutrition, specific, personalized diets, skin nutrients, control of obesity and malnutrition & 48 \\
\hline 9. Nanorobotic tissue rebuilding techniques & 44 \\
\hline 10. Alterations/adjustments in skin and gut microbiota & 41 \\
\hline 11. Immune system manipulations & 33 \\
\hline 12. Auto- and allo- transplantations for cosmetic purposes (other than fat transfers) & 33 \\
\hline
\end{tabular}

search were evaluated by the author and two independent reviewers (non-academic practitioners) in an attempt to identify for this review and summarize the literature on the topic in the context of relevancy to current research and practitioners' interests as affirmed by a survey of practicing board-certified plastic surgeons. These board-certified plastic surgeons participated in the survey ranking the clinical importance (current and future) of twelve issues and their predicted or expected impact on the practice of face and neck rejuvenation. Each respondent ranked the twelve categories by assigning a score " 12 " to the modality expected to exert the most prominent impact in descending fashion, ending on " 1 " given to the treatment with the least expectations. Scores were then totaled and ranked for each of the issues.

Twelve board-certified plastic surgeons were surveyed, ranking areas of current and future research and clinical developments targeting facial and neck aging which will likely have the highest impact on the practice of rejuvenation [Table 1]. The results of the survey indicate that nano and regenerative medical and surgical developments and new technologies are expected to have the largest influence moving forward and reaffirm the hypothesis reflected originally.

\section{COMBINING NANO AND REGENERATIVE TISSUE RESEARCH AND TECHNOLOGIES}

Primary technologies enabling interventions at the molecular level, e.g., prevention of telomere attrition and subsequent protection of DNA and structural proteins of the skin from loss of growth factors and ultimately aging or the use of platelet-rich plasma for skin rejuvenation, are examples of applications of such emerging nanotechnology and regenerative techniques ${ }^{[1,4,9,10]}$.

Nano materials and formulations currently tested in cosmetic medicine include scaffolds for cells and fillers, agent (e.g., growth factors) delivery, applications for cellular modification, isolation and tracking, and nanodevices/robots (e.g., biosensors). Their small size and ability to enter even cell organelles as well as technologies enabling the release of active agents are key ${ }^{[9]}$. Nano systems, nano-assisted cosmetic interventions, and regenerative medicine and surgery have the potential to change facial rejuvenation. Leveraging and enhancement of endogenous stem cells and self-repair mechanisms will likely lead to the development of precision cosmetology (similar to precision medicine) by identification of signaling and effective tissue intercellular substrates and intracellular targets; and current trends, which are generally based on physician intuition and experience, will begin to disappear as palliation of signs of facial and neck aging progress ${ }^{[1]}$. Futuristically, one can imagine a synergy of nano and regenerative systems by cosmeceuticals or stem cell delivery by nanocarriers steering towards a specific target organ, e.g., face or neck, by external magnetic fields and light-triggered release of active agents ${ }^{[12]}$. 

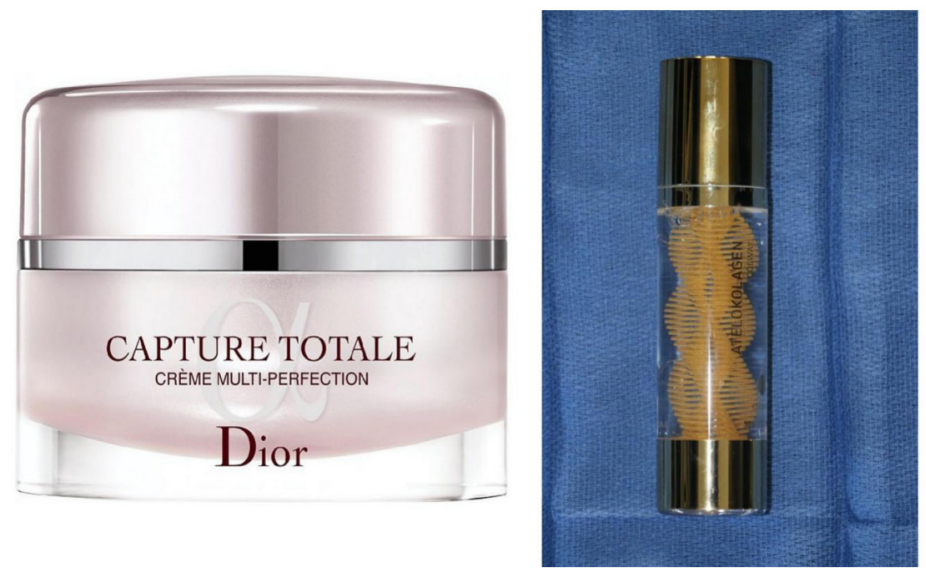

Figure 1. Nano technology for skin care: From "Capture Totale" antiaging cream - skin cosmeceutical utilizing nano-liposomal technology for improved transepidermal and percutaneous delivery introduced by Dior ${ }^{\circledR}$ and representing technology with moistureloaded liposomes which reduce skin water deprivation - to "Atelokolagen" by Colway which contains sericin - capable of carrying both hydrophilic and hydrophobic agents with intracellular uptake

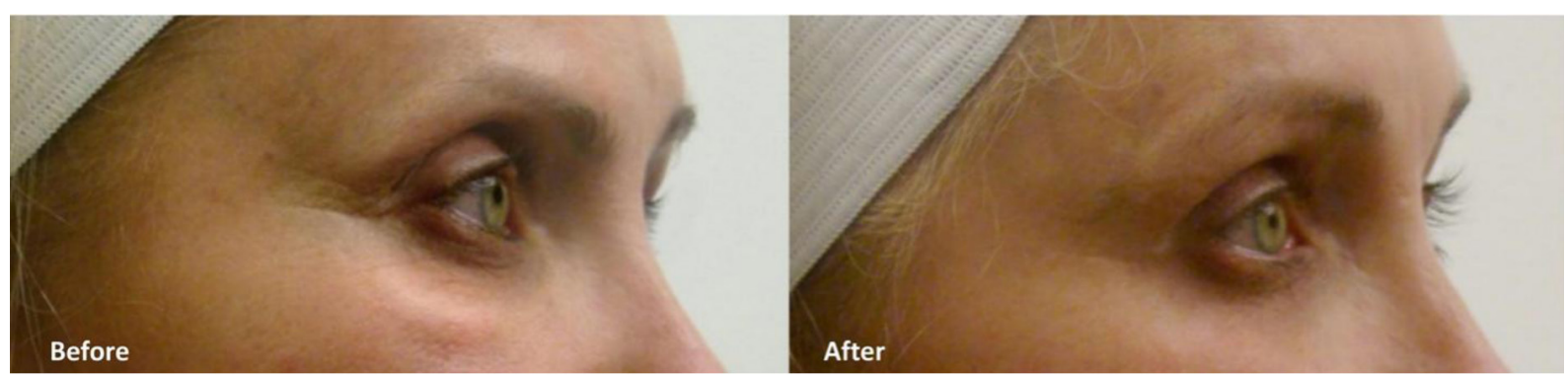

Figure 2. Crows feet skin wrinkles before and after daily application of nano-collagen for 3 weeks (Marine Collagen Biologicals, Inc., San Diego, CA). Results were less spectacular in other cases, however, perhaps due to different individual nuclear gene expression profiles and skin biology ${ }^{[16]}$

Topical and cutaneous delivery of cosmeceuticals evolved from formulations utilizing nano systems to enhance percutaneous delivery of tissue-rejuvenating agents (e.g., the first nano cream Capture Totale developed by Dior ${ }^{\bullet}$, France) to agents with nanocarriers ensuring specific targeted extra- or intracellular cosmeceutical delivery (e.g., sericin containing Atelokolagen developed by Colway, Poland) [Figure 1] $]^{[4,13]}$. Certainly, variability in tissue rejuvenating techniques and agents depends not only on the modality of delivery, including surgery, but also on the pheno- and genotypic characteristics of the subject. Currently, personalized cosmetic selection to effectively target specific features is likely mostly through empiric processes which lead to the establishment of an individualized beauty and rejuvenation "regimen" ${ }^{\text {"[4,15] }}$ [Figure 2].

It is probably only a matter of time until personalized skin care and facial rejuvenation techniques will include interventions on a genotypic level (e.g., by shutting down unfavorable genes) or by triggering regenerative processes ${ }^{[16]}$. These beliefs and modalities were consistently demonstrated and highly ranked within the plastic surgery community ${ }^{[5,16]}$. Transcription factors activate gene supporting processes which lead to cell repair and such factors as forkhead-box protein O3a have a high capacity to preserve skin DNA in younger individuals. As with aging agents, such as sericin, which may be useful to counteract the reduction of cells' capacity for self-repair and dermal regeneration, enhancing the targeted delivery of cosmeceuticals to tissues and even cellular organelles may ultimately reduce and/or reverse skin "decay ${ }^{\text {"1,4,5] }}$ [Figure 3].

Surveyed physicians recognized that there is a growing level of evidence that mesenchymal stem cells from adipose tissue and perivascular cellular components exert a strong potentiating effect on angiogenesis, 


\section{Can we slow down or even reverse aging?}

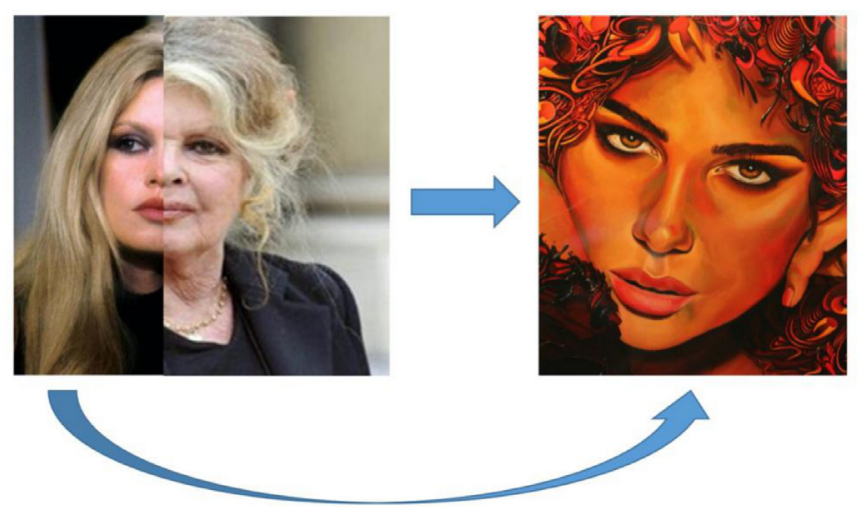

Figure 3. How far are we willing to go to alter, edit, or prevent decay of the skin and/or body genome? Are we able to slow down aging (lower arrow) or reverse skin decay that has already taken place (upper arrow)?

and that they stimulate the regenerative ability of skin by maintaining stem cells that are present in the skin or by assuming the role of native cells upon injection/deposition. Signaling pathways for cellular interactions within the skin and their "controls" appear to be at the core for the maintenance of youthful facial and neck tissues or for the reversal of the loss of a smooth skin surface, dermal cohesion, and/or firmness ${ }^{[2,8]}$. One of the already existing and commercially available systems (SkinMedica TNS Recovery Complex $^{\oplus}$, SkinMedica ${ }^{\oplus}$, An Allergan Company, Anaheim, CA), capable of upregulation of extracellular matrix regeneration controlling genes, exemplifies the feasibility and effectiveness of skin rejuvenation interventions at a molecular level ${ }^{[17]}$.

Technical refinements of tissue replacement by fat grafting are based on at least level III evidence, and they include such factors as the physical form of transferred fat, ratios between the vascularized recipient site and surface/volume of the adipose tissue graft, and the proportion between the stroma and adipocytes ${ }^{[18]}$. Adipose-based tissue graft volumization in combination with customized laser-based skin resurfacing may lead to marked improvements in facial rejuvenation outcomes, as it appears that both modalities may augment specific individual effects ${ }^{[7]}$. Potentiation of the viability, and ultimately the retention rate of the adipose cell grafts by the stromal vascular fraction (including enhancements by the addition of stem cells obtained by culturing), may require more than one operative step. For example, obtaining extra stem cell tissue cultures for 2-3 weeks may be required prior to the addition of stem cells to newly procured adipose tissue. Increased ratio between stem cells (or stroma) and the adipose cells may enhance composite autograft potential, but the entire procedure requires two steps (harvest of adipose tissue to obtain stroma and produce stem cells and procurement of fat to be enriched with cultured stem cells prior to usage $)^{[19]}$.

Therefore, from the standpoint of practicality in cosmetic surgery, tissue regeneration and/or enhancing products which can be stored and "taken off the shelf" - just as many synthetic tissue fillers are - is of particular interest ${ }^{[8,20,21]}$. For example, the mVASC (Microvascular Tissues, Inc., San Diego, CA) product, which consists of aseptically-processed and freeze-dried allogeneic microvascular tissue derived from donated tissue, requires less than one minute to prepare for use. This is in contrast to procedures involving commercially available closed systems for isolating platelet rich plasma, which typically require approximately 30 min to obtain ${ }^{[10]}$. The proprietary mVASC manufacturing process eliminates the proliferative ability of cells within the graft as well as its immunogenicity. The resultant injectable tissue graft (which can also be applicable as a topical agent, e.g., to senescent wounds) is stable at room temperature for four years ${ }^{[20]}$ [Figure 4]. Ongoing trials indicate enormous angiogenic potential of mVASC through upregulation of signaling cascades resulting in tissue remodeling, regeneration, rejuvenation of 


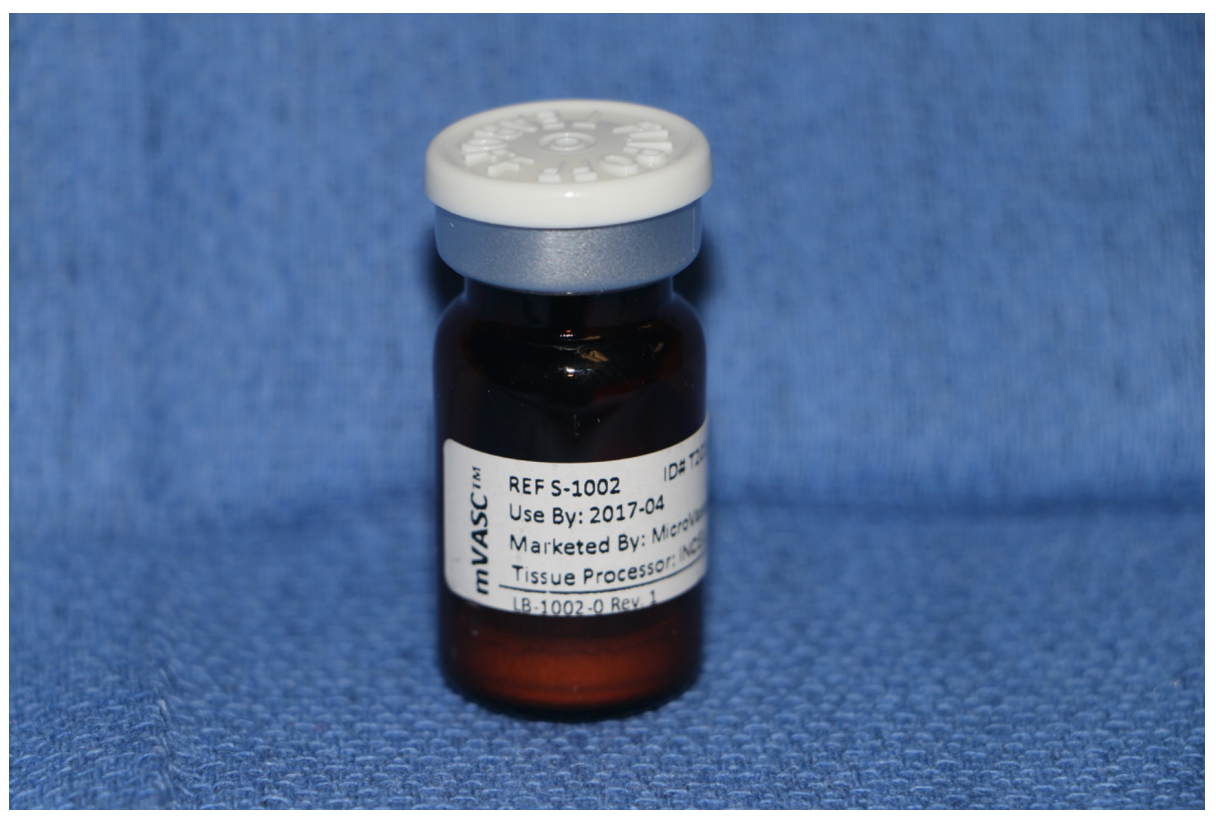

Figure 4. A vial of mVASC with the lyophilized and sterilized pellet containing microvascular tissue fragments. The standardized formulation of mVASC contains an amount of the graft dispersed into one vial such that is ready for dilution, and forms isotonic solution upon the addition of $1 \mathrm{~mL}$ of sterile water

its architecture, and thickness, which - no doubt - will find future aesthetic applications because results of ongoing trials are so encouraging ${ }^{[8,20]}$.

\section{CONCLUSION}

Notably, the survey demonstrated that such modalities as tissue contouring by energy delivering devices, refinements in surgical techniques, alterations in skin and gut microbiota, and transplantation-based (other than adipose tissue) methods were perceived as methods that exert a lesser effect on the future management of facial and neck aging than those "top modalities" including nano and regenerative techniques ${ }^{[16,19,20]}$ [Table 1].

Considering the relatively fast-paced changes and progress in cosmetic surgery and medicine, as well as the novelties that are frequently thrown on the market - oftentimes without prior rigorous clinical studies or trials - suggests that words of caution are appropriate. Recipients of cosmetic care are particularly vulnerable due to the fact that, in many countries, cosmeceuticals are not regulated as rigorously as pharmaceutical drugs, and there is also the risk of addiction to aesthetic procedures. Patients may develop a psychological obsession with repeated or multiple cosmetic procedures and may obsessively seek invasive and noninvasive aesthetic novelties. These patients lose objectivity and criticism, and oftentimes become victims of unscrupulous providers. Even those who observe safety issues may not appreciate the importance of the fact that there is a separate categorization of the quality of evidence and strength of recommendations available ${ }^{[22,23]}$. Failure to recognize the quality of evidence can lead to misguided recommendations. The assessment of trends and developments in facial and neck rejuvenation requires particular attention to the quality of research available prior to its clinical application, as emerging evidence includes an element of uncertainty about tradeoffs - the balance among safety and desirable and undesirable effects is not welldefined. With the inevitability of scientific progress, this new level of knowledge regarding the variability in care responses and indications for procedures on almost a "molecular level" will likely lead to personalized and precise aesthetic surgery and medicine becoming a nearby reality. 


\section{DECLARATIONS}

\section{Authors' contributions}

Made substantial contributions to conception and design of the study and performed data analysis and interpretation: Dobke M, Hauch A

\section{Availability of data and materials}

Not applicable.

\section{Financial support and sponsorship}

None.

\section{Conflicts of interest}

All authors declared that there are no conflicts of interest.

\section{Ethical approval and consent to participate}

Not applicable.

\section{Consent for publication}

Not applicable.

\section{Copyright}

(c) The Author(s) 2020.

\section{REFERENCES}

1. Lohani A, Verma A, Joshi H, Yadav N, Karki N. Nanotechnology-based cosmeceuticals. ISRN Dermatol 2014;2014:843687.

2. Gaur M, Dobke M, Lunyak VV. Mesenchymal stem cells from adipose tissue in clinical applications for dermatological applications and skin aging. Int J Mol Sci 2017;18:1-29.

3. Dobke M, Sikora NC, Vargas F. Skin aging and beauty exploring the gut microbiota connection. J Aesth Rec Surg 2019;5:1-3.

4. Mandal BB, Kundu SC. Self-assembled silk sericin/poloxamer nanoparticles are nanocarriers of hydrophobic and hydrophilic drugs for targeted delivery. Nanotechnology 2009;20:355101.

5. Goldfaden R, Goldfaden G. Target wrinkle formation with novel peptides. Life Extension 2014. Available from: https://www.lifeextension. com/magazine/2014/4/target-wrinkle-formation-with-novel-peptides [Last accessed on 10 Jan 2020]

6. Limongi T, Canta M, Racca L, Ancona A, Tritta S, et al. Improving dispersal of therapeutic nanoparticles in the human body. Nanomedicine (Lond) 2019;14:797-801.

7. Cohen SR, Womack H. Injectable tissue replacement and regeneration: anatomic fat grafting to restore decayed facial tissues. Plast Reconstr Surg Glob Open 2019;7:1-11.

8. Terlizzi V, Kolibabka M, Burgess JK, Hammes HP, Hamsen MC. The pericytic phenotype of adipose tissue-derived stromal cells is promoted by NOTCH2. Stem Cells 2018;36:240-51.

9. Prow TW, Grice JW, Lin LL, Faye R, Butler M, et al. Nanoparticles and microparticles for skin drug delivery. Adv Drug Deliv Rev 2011;63:470-91.

10. Samadi P, Sheykhhasan M, Khoshinani HM. The use of platelet-rich plasma in aesthetic and regenerative medicine: a comprehensive review. Aesthetic Plast Surg 2019;43:803-14.

11. Verma S, Domb A, Kumar N. Nanomaterials for regenerative medicine. Nanomedicine (Lond) 2011;6:157-81.

12. Jin Z, Ngyuen KT, Go G, Kang B, Min HK, et al. Multifunctional nanorobot systems for active therapeutic delivery and synergistic chemo-photothermal therapy. Nano Lett 2019;19:8550-64.

13. Dominguez S, Mackert GA, Dobke M. Nanotechnology to enhance transdermal delivery of hydrophilic humectants for improved skin care: a model for therapeutic applications. In: Andronescu E, Grumezescu AM, editors. Nanostructures for drug delivery. Amsterdam: Elsevier; 2017. pp. 919-39.

14. Sikora NC, Dobke M. Postmenopausal skin aging - exploring the significance of estrogen-gut microbiota axis. JJ Expt Derm 2019;3:1-4.

15. Dobke M, Sikora NC, Vargas F. Skin aging and beauty exploring the gut microbiota connection. J Aesthet Reconstr Surg 2019;5:1-3.

16. Velmeshev D. Single-cell genomics in disease research and diagnostics. Clin Lab Manager 2019;4:18-21.

17. Kadoya K, Makino ET, Jiang LI, Bachelor M, Chung R, et al. Upregulation of extracellular matrix genes corroborates clinical efficacy of human fibroblast-derived growth factors in skin rejuvenation. J Drugs Dermatol 2017;16:1190-6.

18. Cohen SR, Womack H. Injectable tissue replacement and regeneration: anatomic fat grafting to restore decayed facial tissues. Plast Reconstr Surg Glob Open 2019;7:e2293. 
19. Park JY. Stem cell fat graft. In: Liposuction. Singapore; Springer; 2018. pp. 253-74.

20. Dobke M, Berger JS. Allogeneic microvascular tissue graft restores healing capacity in recalcitrant cleft palate fistula: a case report. J Den Max Surg 2019;2:164-70.

21. Raper V. Gene therapies are being brought to a wider audience. Genet Eng Biotechnol News 2019;39:7-9.

22. Guyatt GH, Oxman AD, Vist GE, Kunz R, Falck-Ytter Y, et al. GRADE: an emerging consensus on rating quality of evidence and strength of recommendations. BMJ 2008;336:924-6.

23. Leask F. Spotting 'unproven' stem cell therapies in the wild. Biotechniques 2019;67:253-5. 